\title{
Effects of reinforcement on retention and transfer: Pattern discrimination
}

\author{
ELVIS C. JONES AND RICHARD L. NARVER
}

TEXAS CHRISTIAN UNIVERSITY

In two experiments, Ss learned to discriminate between patterns drawn from different pattern populations. Different groups received consistent, intermittent, or no extrinsic reinforcement. The groups which received extrinsic reinforcement tended to remember pattern characteristics, and demonstrated transfer to a new task, better than did the unreinforced groups. Results were generally consistent with an earlier experiment which indicated that monetary rewards improve recall, relearning, and transfer in a verbal learning task.

Jones, Edmonds, \& Mueller (1966), attempted to determine if reinforcement affects resistance to forgetting as well as persistence of responding during extinction. They found that extrinsic reinforcement during acquisition resulted in better recall, relearning, and transfer of rote serial learning. The effects of partial reinforcement were significantly different from consistent reinforcement in transfer, but not in recall or relearning. The reinforcer was money.

The present pair of experiments represented an attempt to determine if a different reinforcer (knowledge of results) can produce the same effects in a different task (pattern discrimination) using a different retention measure (identification). Experiment I attempted to determine if consistent and intermittent knowledge of results during acquisition produces better retention of the common characteristics of patterns, and better transfer to a new discrimination task. Experiment II involved a shorter retention period and assessed the effects of an association between the correct discrimination response and the presence of a relatively meaningless symbol.

\section{METHOD}

\section{Experiment l.}

The Ss were 30 students enrolled in an introductory psychology class, randomly assigned to three equal groups. Training materials consisted of 20 sets of patterns, three patterns per set. Each set was printed on a separate page of a booklet. Two patterns in each set were randomly drawn from one population of patterns (the "common" population), and one pattern in each set was drawn from another population (the "odd" population). Each pattern consisted of a histoform with 12 columns of zeros. Each column, within the population, had a normal distribution of heights, with a mean between 4 and 11 zeros and a standard deviation of one. The population mean column heights of the individual columns were: $6,11,9,7$, $10,5,11,9,7,4,10,11$, for the common population, and: $8,9,10,6,10,10,10,10,8,6,9,8$, for the odd population. The populations were intentionally constructed so as to be difficult to distinguish. The positions of the patterns within the booklet sets were randomized to prevent position learning.

In the booklets given to one group, the number of the odd pattern was printed on the reverse of every sheet (CR group). In the booklets given to a second group, the number of the odd pattern was printed on the reverse of only $50 \%$ of the sheets, randomly determined ( $P R$ group). In the booklets given to the third group, numbers were not printed on the reverse of any sheet (NR group).

The test booklets contained six sets of the three patterns. Three sets consisted of one pattern drawn from the common population and two patterns with randomly determined column heights. There were also three sets of patterns which consisted of one pattern drawn from the odd population and two with randomly determined column heights. There were no numbers on the backs of any of the sheets. The six test sets were randomly arranged to balance for any learning that occurred during testing.

After receiving the training booklets, the Ss were instructed to look at each set of patterns and decide which pattern was most unlike the other two. They were further instructed to mark that pattern within 25 sec., then turn the sheet over and check the answer on the reverse. They were also told that not all sets had answers, and, after checking and finding no answer, they were to go on to the next set without delay.

Ss were not told that they would later be tested. Two days after the training session, all Ss were given the test booklet and told to check the pattern in each set which was most similar to the patterns encountered during the training session. They were told that none of the test sets had answers on their reverses, and were allowed $25 \mathrm{sec}$. to finish each set.

\section{Experiment II.}

Experiment II differed from Experiment I only in the following ways: Two groups were used. The numbers of all three patterns were printed on the reverse of each sheet in the training booklets, and each number was accompanied by one of three symbols $(\cdot+*)$. In the booklets given to one group (R group), the symbol $(\cdot)$ always accompanied the number of the odd pattern, and the two other symbols were randomly assigned to the two common patterns. In the booklets given to the other group (NR group), all symbols were randomly assigned to the pattern numbers. The same test 
booklets as in Experiment I were used, but the test was given within $5 \mathrm{~min}$. following completion of the training booklets.

\section{RESULTS AND DISCUSSION}

Although the learning task was difficult, Ss did eventually learn to consistently identify the odd patterns. The reinforced groups learned more rapidly than did the unreinforced groups, but there was no difference in performance at the end of training.

The test of recall consisted of performance on the first test trial. All groups in Experiment I performed at, or below, chance level. Therefore, no comparisons between groups were made. Apparently, after two days, all Ss had forgotten to such a degree that group differences would be meaningless. The transfer task consisted of performance over the subsequent trials. The reinforced groups in Experiment I were superior to the NR group $(t=2.15, d f=28, p<.025)$. There was, however, no difference between the CR and PR groups.

In Experiment II, with a short retention period, both groups performed above chance level on recall. The $\mathrm{R}$ group performed better than did the NR group (Fisher-Yates exact probability test, $\mathrm{p}<.05$ ). However, both groups performed without error on subsequent trials, making a comparison of transfer meaningless. Apparently, the retention period was too short for the purpose of measuring transfer effects.

There was no significant difference during testing, in either experiment, between the ability to identify patterns drawn from the odd vs. the common populations. Both groups in Experiment II performed better during testing than they had during training, suggesting that while there was difficulty in discriminating between patterns, Ss nevertheless learned the characteristics that all of the patterns had in common.

In Experiment I, reinforcement was in the form of direct knowledge of results, whereas in Experiment II, reinforcement was a function of the contingency between marking a specific pattern and finding that the same, relatively meaningless, symbol was consistently associated with the marked pattern. Since the Ss in Experiment II were told only to look at the pattern numbers and symbols, and since they were not told which, if any, symbol indicated the correct response, it is difficult to explain why the symbols operated as reinforcers. One possibility is that during problem solving, practically any event that consistently follows a specific response may be interpreted as confirmation of the correctness of the response. Were this the case, we would have the reverse of typical superstitious learning. Rather than learning a response which spuriously precedes reinforcement, in this case, neutral stimuli would become reinforcing if they spuriously followed certain responses.

The Es feel that the present results would have been more consistent with the serial learning study if the retention periods had been shorter than in Experiment I, but longer than in Experiment II. In agreement, for the most part, with Jones, Edmonds, \& Mueller (1966), the data indicate that reinforcement increases resistance to forgetting and enhances transfer to a new task.

\section{Reference}

Jones, E. C., Edmonds, E. M., \& Mueller, M. R. Effects of reinforcement on retention and transfer. Psychon. Sci., 1966, 6, 181-182. 